\title{
Downregulation of miR-182-5p inhibits the proliferation and invasion of triple-negative breast cancer cells through regulating TLR4/NF-кB pathway activity by targeting FBXW7
}

\author{
Xingping Wu, Hao Chen, Miantao Wu, Songguo Peng, Lin Zhang \\ State Key Laboratory of Oncology in South China, Sun Yat-sen University Cancer Center, Guangzhou, China \\ Contributions: (I) Conception and design: X Wu, L Zhang; (II) Administrative support: H Chen, M Wu, S Peng; (III) Provision of study materials \\ or patients: H Chen, M Wu, S Peng; (IV) Collection and assembly of data: H Chen, M Wu, S Peng; (V) Data analysis and interpretation: X Wu, L \\ Zhang; (VI) Manuscript writing: All authors; (VII) Final approval of manuscript: All authors. \\ Correspondence to: Xingping Wu; Lin Zhang. State Key Laboratory of Oncology in South China, Sun Yat-sen University Cancer Center, 651 \\ Dongfeng Road East, Guangzhou, China; Email: wuxp@sysucc.org.cn; zhanglin@sysucc.org.cn.
}

\begin{abstract}
Background: To investigate the role of miR-182-5p in the proliferation and invasion of triple-negative breast cancer (TNBC) cells, as well as the underlying mechanism.

Methods: qRT-PCR was used to detect the level of miR-182-5p in tissues and cells. CCK-8 assay, flow cytometry, and Transwell assay were performed to detect cell proliferation, apoptosis rate, and invasion, respectively. Pearson correlation analysis was used to determine the correlation between miR-182-5p and its predicted target gene FBXW7, and the target of miR-182-5p was identified by dual-luciferase reporter gene assay. ELISA assay was used to examine the levels of cytokines in the culture supernatant of tumor cells. Western blot analysis was used to detect the expressions of FBXW7, TLR4, and NF- $\mathrm{B}$ ) pathways in tumor cells.

Results: In MDA-MB-231 and BT-549 cells, downregulation of miR-182-5p significantly inhibited cell proliferation and invasion and promoted tumor cell apoptosis. Pearson correlation analysis showed that miR182-5p had a negative correlation with FBXW7. Dual-luciferase reporter gene assay showed that miR-1825p could directly target FBXW7. Further studies showed that FBXW7 overexpression significantly inhibited cell proliferation and invasion and increased the apoptosis rate. Downregulation of miR-182-5p significantly reduced the levels of TNF- $\alpha, \mathrm{IL}-1 \beta$, IL- 6 , and IL-18 in the culture supernatant, and decreased the activity of TLR4/NF- $\kappa B$ pathway in tumor cells, while downregulation of FBXW7 significantly inhibited the effect of miR-182-5p on tumor cells.
\end{abstract}

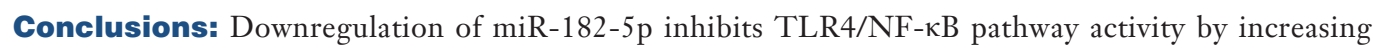
FBXW7 expression, thereby suppressing the proliferation and invasion of TNBC cells.

Keywords: miR-182-5p; triple-negative breast cancer (TNBC); proliferation; invasion; TLR4/NF-кB pathway

Submitted Jun 12, 2020. Accepted for publication Jul 29, 2020.

doi: $10.21037 /$ atm-20-5192

View this article at: http://dx.doi.org/10.21037/atm-20-5192

\section{Introduction}

Breast cancer (BC), the most common cancer among women in the world, can be divided into many molecular subtypes, including Luminal A, Luminal B, human epidermal growth factor receptor 2 (HER2)-overexpressing, normal breast-like and basal-like or triple-negative breast cancer (TNBC). TNBC is a BC subtype that does not express the estrogen receptor (ER), progesterone receptor (PR), and HER2. TNBC can be further classified into six subtypes, including immunomodulatory, mesenchymal, mesenchymal stem-like, luminal androgen receptor, basallike 1, and basal-like 2. The total incidence rate of TNBC 
is about $15-20 \%$. Compared with hormone receptor and HER2 positive BC, TNBC has a highly invasive clinical process, with earlier onset age, more significant metastatic potential, poorer clinical prognosis, higher recurrence rate, and lower survival rate $(1,2)$. The traditional treatment of TNBC mainly includes adjuvant therapy, surgical treatment and radiotherapy. Adjuvant therapy is a key strategy for cancer treatment, which can avoid the risk of metastasis accompanied by rapid progression and tumor recurrence. However, chemoresistance is a major problem in adjuvant treatment of cancer, with a failure rate of up to $90 \%$ in the treatment of metastatic cancer (3). In addition, TNBC is not sensitive to endocrine therapy or molecular targeted therapy because of its special molecular phenotype. Therefore, systemic chemotherapy is still the main means of treatment, but conventional postoperative adjuvant radiotherapy and chemotherapy has poor efficacy, residual metastases will eventually lead to tumor recurrence, and more side effects (4). However, there is no approved targeted therapy strategy for TNBC. Systemic chemotherapy is still the primary method of treatment, and the therapeutic effect of chemotherapy often lasts for a short time, causing the treatment of TNBC to be a clinical challenge (4). Therefore, the development of more effective treatment strategies is of great significance for the treatment of TNBC.

Increasing evidence showed that inflammation contributes to the occurrence of tumors. Inflammatory cells can promote tumor formation, release survival factors, promote angiogenesis and lymphangiogenesis, stimulate DNA damage, reshape the extracellular matrix to promote invasion, coat tumor cells to provide receptors that transmit cells through lymphatics and capillaries, and evade host defense mechanisms (5). Toll-like receptor 4 (TLR4), one of the essential receptors on the surface of immune cells, is often expressed in tumor cells and participates in the progression, invasion, and drug resistance of $\mathrm{BC}(6,7)$. TLR4 activates myeloid differentiation factor 88 (MyD88) after receiving tumor antigen information, promotes the nuclear transfer of nuclear factor- $\kappa \mathrm{B}(\mathrm{NF}-\kappa \mathrm{B})$, and activates gene transcription, inducing the production of proinflammatory cytokines and causing inflammatory reactions $(8,9)$. According to research reports, NF- $\mathrm{BB}$ pathway may be a key regulator in the progress of TNBC (10), which can participate in the growth, apoptosis, differentiation and inflammatory response of tumor cells by promoting the transcription of tumor regulatory factors, including cytokines, chemokines, cell adhesion molecules, and inducible pro-inflammatory enzymes, and ultimately affect the invasion and progress of tumor (11-14).

microRNAs (miRNAs), a type of small non-coding RNA composed of 18-24 single nucleotides, participates in the tumorigenesis process of various cancers, including BC (15). For instance, miR-185-5p inhibits invasion, migration, and epithelial-mesenchymal transition by regulating the specific receptor for advanced glycation end products in BC. MiR-19b can be used as a prognostic biomarker for BC and can promote cell proliferation, migration, and invasion by mediating phosphatidylinositol 3-kinase and serinethreonine kinase pathways (16). MiR-182, a miRNA with dual functions, is found on human chromosome $7 \mathrm{q} 32.2$ in the miR-183/96/182 cluster. It has been proved that miR182 is up-regulated in TNBC, melanoma, endometrium, colon, and prostate and plays a tumor inhibitory role in lung cancer and gastric cancer (17-19). Also, studies have revealed that $\mathrm{miR}-182-5 \mathrm{p}$ promotes the invasion and proliferation of prostate cancer cells by mediating forkhead protein F2, reversal inducting cysteine-rich Kazakh motif protein, and transferring inhibitor 1 to promote the invasion and proliferation of prostate cancer cells (20). Inhibition of miR-182-5p can induce apoptosis and antiproliferation by regulating the expression of Caspase 9 in BC (18). Recent studies have shown that down-regulation of mir-182-5p can inhibit the proliferation and invasion of breast cancer by targeting the phosphatase tensin homologous gene (PTEN) deleted on chromosome 10 (21). However, the role and potential molecular mechanism of mir-182-5p in TNBC are still unclear.

In this study, we hypothesized that inhibition of miR$182-5 \mathrm{p}$ could take part in the regulation of TNBC cell proliferation and invasion by downregulating TLR4/NF$\kappa \mathrm{B}$ inflammatory pathway activity. For this purpose, we evaluated the effect of miR-182-5p down-regulation on TNBC cell proliferation, apoptosis, and invasion as well as its possible mechanism by performing miR-182-5p loss of function experiment.

We present the following article in accordance with the MDAR reporting checklist (available at http://dx.doi. org/10.21037/atm-20-5192).

\section{Methods}

\section{Materials}

The tissue samples and adjacent normal tissues of 50 patients with TNBC treated in State Key Laboratory 
Table 1 qRT-PCR primer sequences

\begin{tabular}{lll}
\hline Gene & Primer & Sequence \\
\hline miR-182-5p & $\mathrm{F}$ & 5'-TGCGGTTTG GCAATGGTAGAAC-3' \\
& $\mathrm{R}$ & 5'- CCA GTGCAGGGTCCGAG GT-3' \\
FBXW7 & $\mathrm{F}$ & 5'-ACTGGAAAGTGAC TCTGGGA-3' \\
& $\mathrm{R}$ & 5'-TACTGGGGCTAGGCAAACAA-3' \\
U6 & $\mathrm{F}$ & 5'-TTATGGGTCCTAGCCTGAC-3' \\
& $\mathrm{R}$ & 5'-CACTATTGCGGGCTGC-3' \\
GAPDH & $\mathrm{F}$ & 5'-ACAGCAACAGGGTGGTGGAC-3' \\
& $\mathrm{R}$ & 5'-TTTGAGGGTGCAGCGAACTT-3'
\end{tabular}

of Oncology in South China, Sun Yat-sen University Cancer Center from January 2017 to December 2019 were collected, and the basic information of patients including age, gender, blood pressure, tumor stage, whether there was distal metastasis and whether there was lymph metastasis was recorded. Normal human breast cells MCF10A, TNBC cells MDA-MB-231, MDA-MB-453, MDA-MB-361, and BT-549 were purchased from Shanghai Cell Bank of the Chinese Academy of Sciences (Shanghai, China). This study was approved by the Ethics Committee of Sun Yatsen University Cancer Center (No. RDDA2020001590).

\section{Cell culture and transfection}

Cells were cultured in DMEM medium supplemented with $10 \%$ fetal bovine serum (FBS), $100 \mathrm{mg} / \mathrm{mL}$ penicillin, and $100 \mathrm{U} / \mathrm{mL}$ streptomycin in a constant temperature incubator at $37{ }^{\circ} \mathrm{C}, 5 \% \mathrm{CO}_{2}$, saturation humidity. Cell transfection: MDA-MB-231 and BT-549 cells were selected for miR-182-5p loss of function experiment. Experiment 1: cells were divided into negative control group (NC) and miR-182-5p loss of function group (miR-182-5p inhibitor). The medium was replaced with a serum-free medium that did not have penicillin and streptomycin during transfection. When cell culture reached about $70 \%$ confluence, miR-182-5p inhibitor NC or miR182-5p inhibitor are transfected into MDA-MB-231 or BT-549 cells respectively in line with the description of Lipofectamine ${ }^{\mathrm{TM}}$ reagent. Experiment 2: the cells were divided into two groups: negative control group (pcNC) and FBXW overexpression group (FBXW7). When the fusion degree of cell culture reached about $70 \%$, the overexpression plasmids of pc-NC and pcDNA3.1-FBXW7 were transfected into MDA-MB-231 or BT-549 cells in the same way as above. Experiment 3: cells were divided into empty-NC group (pc-NC), FBXW7 overexpression group (FBXW7), and miR-182-5p inhibitor + FBXW7 interference group (miR-182-5p inhibitor + si-FBXW7). When the fusion degree of cell culture reached about $70 \%$, miR-182-5p inhibitor NC or miR-182-5p inhibitor and siFBXW7 plasmids were transfected into MDA-MB-231 or BT-549 cells in the same way. The above plasmids of miR182-5p inhibitor/NC, pcDNA3.1, pc-NC and si-FBXW7/ si-NC plasmids were designed and completed by Sangon Biotech (Shanghai) Co., Ltd. (Shanghai, China). After transfection for 6-8 hours, the culture medium was replaced by normal complete medium.

\section{$q R T-P C R$ to detect miR-182-5p and FBXW7 expression}

Trizol kit (Invitrogen, CA, USA) was employed to extract total RNA in cells or tissues. The concentration of RNA was determined by NanoDrop 2000 spectrophotometer (Thermo Fisher Scientific, USA). MiRNA was reverse transcribed into cDNA utilizing TaqMan miRNA reverse transcription kit (Thermo Fisher Scientific, USA). The expression level of miR-182-5p was determined by TaqMan miRNA detection kit (Thermo Fisher Scientific, USA). PrimeScript ${ }^{\mathrm{TM}}$ RT (Takara, Japan) was used for reverse transcription to synthesize cDNA of mRNA. PCR amplification was performed by $\mathrm{SYBR}^{\circledR}$ Green Real-time PCR-Master Mix (Toyobo, Japan) to detect the level of FBXW7. The expression of miR-182-5p and FBXW7 were normalized with U6 and GADPH as internal parameters, respectively, and the relative expression was calculated using the $2^{-\triangle \Delta C t}$ method. QRT-PCR reaction conditions: pre-denaturation at $95{ }^{\circ} \mathrm{C}$ for $10 \mathrm{~min}$, then 45 cycles of incubation at $95{ }^{\circ} \mathrm{C}$ for $15 \mathrm{~s}$ and $60{ }^{\circ} \mathrm{C}$ for $60 \mathrm{~s}$. The experiment was repeated three times. The primer sequences of the target gene were shown in Table 1.

\section{Detection of cell proliferation, apoptosis, and invasion}

Cell counting kit-8 (CCK-8; Dojindo, Japan) was adopted to detect cell proliferation. Stably transfected cells were collected and seeded into 96-well plates at a density of $1 \times 10^{4}$ cells per well. After culturing for 24,48 , or 72 hours respectively, cells were treated with $10 \mu \mathrm{L}$ CCK- 8 reagent for 2 hours. The absorbance was measured at $450 \mathrm{~nm}$ by enzyme immunoassays (Bio-Rad Laboratories, CA, USA), and the cell proliferation ability was determined. 
Flow cytometry detected the apoptosis level. Cells in the logarithmic phase were collected and separated by trypsin to prepare cell suspension. Then, the cells were fixed overnight with cold ethanol at $4{ }^{\circ} \mathrm{C}$, centrifuged for 5 minutes, and incubated with $5 \mu \mathrm{L}$ of annexin $\mathrm{V}$ - FITC. After $3 \mathrm{~min}$, the cells were supplemented with $10 \mu \mathrm{L}$ propidium iodide (PI), incubated at $37^{\circ} \mathrm{C}$ for $15 \mathrm{~min}$, centrifuged, and resuspended in $0.5 \mathrm{~mL}$ precooled buffer. Flow cytometry (BD Biosciences, NJ, USA) was used to observe the apoptosis.

A Transwell experiment was used to detect cell invasion ability. MDA-MB-231 and BT-549 cells were resuspended in FBS-free DMEM and inoculated into the upper cavity of precoated matrix gel (Becton-Dickinson, CA, USA) for cell invasion analysis. DMEM medium holding $10 \%$ FBS was added to the lower chamber. After incubation for 24 hours, the remaining cells on the upper chamber were wiped off with cotton swabs. Cells infiltrated into the basal surface of the membrane were fixed with $4 \%$ paraformaldehyde, stained with $0.1 \%$ crystal violet after $10 \mathrm{~min}$, and finally, the cells in 5 randomly selected fields were counted under a microscope (Olympus, Japan).

\section{Double luciferase reporter gene experiment}

The binding sites of miR-182-5p and FBXW7 were predicted by using the bioinformatics website (http://www. targetscan.org). FBXW7 3'-UTR promoter region sequence containing miR-182-5p binding site was synthesized, and FBXW7-WT and its corresponding mutation binding site FBXW7-MUT were constructed. MDA-MB-231 and BT549 cells in the logarithmic growth phase were inoculated onto 96-well plates. When cell confluence reached $70 \%$, mimics NC or miR-182-5p mimics, FBXW7-WT and FBXW7-MUT were co-transfected into MDA-MB-231 and MCF-7 cells. Forty-eight hours after transfection, cell lysis was performed, and luciferase activity was measured by luciferase detection kit.

\section{ELISA to detect the levels of TNF- $\alpha, I L-18, I L-1 \beta$, and $I L-6$}

The levels of TNF- $\alpha$, IL-18, IL-1 $\beta$, and IL- 6 in the supernatant of cell culture were determined by ELISA kits following the instructions. Streptavidin-HRP and tetramethylbenzidine sulfonate were used as color development indicators. ELISA microplate reader DR200Bs (Jinan Laibao Laboratory Equipment Co., Ltd., Jinan, China) is used to read the values at $450 \mathrm{~nm}$ wavelengths, and the concentration level of corresponding cytokines is calculated through standard curves.

\section{Western blot to detect the protein expression of TLR4, $M y D 88, N F-\kappa B$, and I- $\mathrm{KB}$}

RIPA buffer (Sigma-Aldrich, MO, USA) containing protease inhibitor was used to split and extract total protein on ice, followed by centrifugation at $4{ }^{\circ} \mathrm{C}$ for $15 \mathrm{~min}$ after $40 \mathrm{~min}$. The protein concentration was then measured using a BCA protein assay kit (Beyotime Biotechnology, Shanghai, China). SDS-PAGE separated the protein in $12 \%$ separation gel, and $4 \%$ concentrated gel and transferred to PVDF membrane. PVDF membrane carrying transfer protein is sealed in TBST buffer solution containing $0.1 \%$ Tween 20 and $5 \%$ bovine serum albumin (BSA) for 2 hours at normal temperature. Subsequently, the designated primary antibody (rabbit anti-FBXW7 antibody $(1: 1,000)$, rabbit anti-TLR4 antibody $(1: 1,000)$, rabbit antiMyD88 antibody $(1: 1,000)$, rabbit anti-p-P65 antibody $(1: 1,000)$, rabbit anti-P65 antibody $(1: 1,000)$, rabbit anti-pI-kappa $b$ antibody $(1: 1,000)$, rabbit anti-I-kappa $b$ antibody $(1: 1,000)$, and mouse anti-GAPDH antibody $(1: 5,000)$ were incubated overnight at $4{ }^{\circ} \mathrm{C}$. Then, the membrane was incubated with the corresponding secondary antibody for 2 hours. After enhanced chemiluminescence and exposure color development, the gray values of protein bands were analyzed by ImageJ software.

\section{Statistical analysis}

SPSS 22.0 software was used to process the data, and the results were expressed by the mean \pm standard deviation (SD) of $\mathrm{n}$ experiments. $T$-test was used to compare the two groups. Multiple comparisons between groups were conducted by one-way analysis of variance (ANOVA), followed by LSD (least significant difference) post hoc test, with $\mathrm{P}<0.05$ showing significant difference.

\section{Results}

\section{MiR-182-5p is bighly expressed in TNBC}

To determine the relationship between miR-182-5p and TNBC, we detected the expression level of miR-182-5p in TNBC tissues and cells by qRT-PCR. The results revealed that the expression level of miR-182-5p in the tumor group was significantly higher than that in the normal group (Figure 1A). Whereas, the expression level of miR-182-5p 

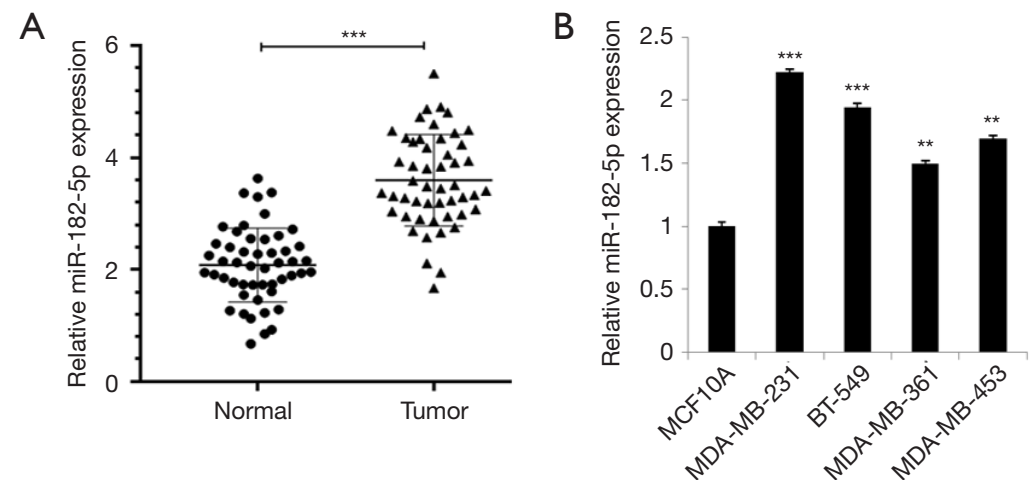

Figure 1 The expression of miR-182-5p in TNBC tissues and cells. (A) The expression of miR-182-5p in TNBC tissues. (B) The expression of miR-182-5p in TNBC cells. **, $\mathrm{P}<0.01$; ${ }^{* * *}, \mathrm{P}<0.001$. TNBC, triple-negative breast cancer.

in TNBC cells was significantly higher than that in normal MCF10A cells, and the expression level was highest in MDA-MB-231 and BT-549 cells (Figure 1B). The above results suggested that miR-182-5p may contribute to the progress of TNBC.

\section{Downregulation of miR-182-5p expression inbibits TNBC cell proliferation and invasion and promotes cell apoptosis}

To further determine the role of miR-182-5p in TNBC, we evaluated the effect of miR-182-5p silencing on TNBC cell proliferation, apoptosis, and invasion through function loss experiment. According to the results in Figure 1B, MDA-MB-231 and BT-549 cells with the highest expression level of miR-182-5p were selected as the research objects of the follow-up experiment. CCK8 results manifested that downregulation of miR-182-5p expression significantly inhibited the cell proliferation ability of MDA-MB-231 (Figure 2A) and BT-549 cells (Figure $2 B$ ), and the inhibition became more evident with the prolongation of action time. Flow cytometry results evinced that downregulation of miR-182-5p expression significantly increased the apoptosis rate of MDA-MB-231 (Figure 2C) and BT-549 cells (Figure 2D). Transwell results verified that the downregulation of miR-182-5p expression significantly inhibited the invasion rate of MDA-MB-231 (Figure 2E) and BT-549 cells (Figure 2F). The above results suggested that miR-182-5p may contribute to TNBC cell proliferation and invasion and inhibit TNBC cell apoptosis.

\section{MiR-182-5p targets FBXW7}

The target of miR-182-5p was predicted through
microRNA.org and Targetscan online tools. FBXW7 scored the highest among all potential targets was found. We first detected the expression level of FBXW7 in TNBC tissue. QRT-PCR results identified that the expression level of FBXW7 in TNBC tissues was significantly lower than that in normal tissues (Figure 3A). Pearson correlation analysis suggested that the expression of miR-182-5p and FBXW7 in TNBC tissue was significantly negatively correlated (Figure 3B). Moreover, we constructed FBXW7 3'-UTR-WT and FBXW7 3'-UTR-MUT luciferase reporter plasmids (Figure 3C). After co-transfecting FBXW7 3'-UTR-WT or 3'-UTR-MUT and miR-182-5p mimics or NC into TNBC cells, we found that miR-182-5p mimic significantly reduced luciferase activity in the cells through double luciferin reporter gene experiments (Figure 3D). Further Western Blot tests indicated that downregulation of miR-182-5p expression in TNBC cells markedly increased FBXW expression (Figure 3E). The above results confirmed that miR-182-5p could target binding and negatively regulate FBXW7.

\section{Overexpression of FBXW7 inbibits proliferation and invasion of TNBC cells and promotes apoptosis}

We further discussed the effects of FBXW7 on the proliferation, apoptosis, and invasion of TNBC cells. CCK8 results suggested that overexpression of FBXW7 significantly inhibited the cell proliferation of MDAMB-231 (Figure 4A) and BT-549 cells (Figure 4B), and the inhibition became more evident with the prolongation of the action time. Flow cytometry indicated that overexpression of FBXW7 remarkably increased the apoptosis rate of MDA-MB-231 (Figure 4C) and BT- 


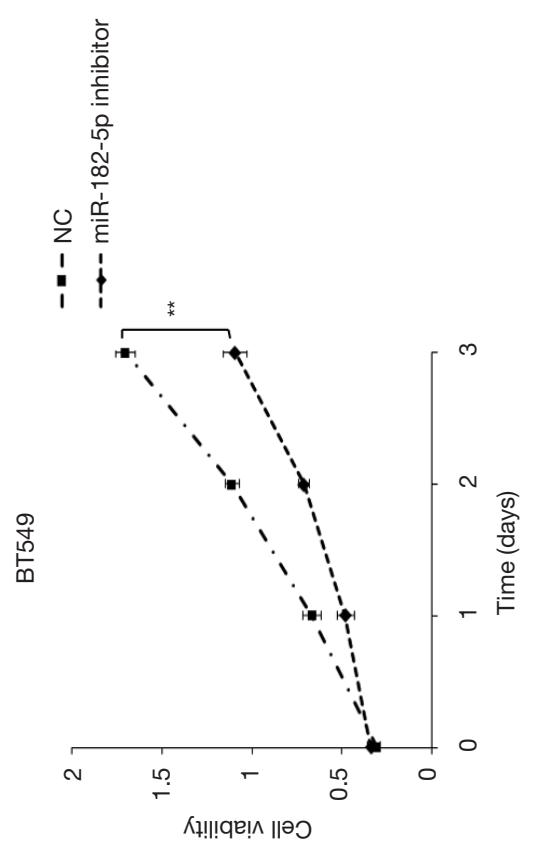

$\infty$

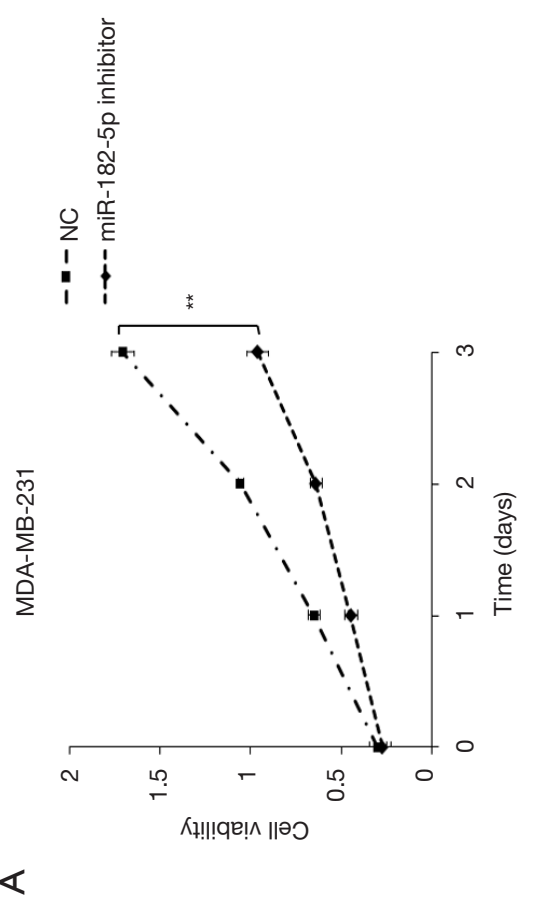

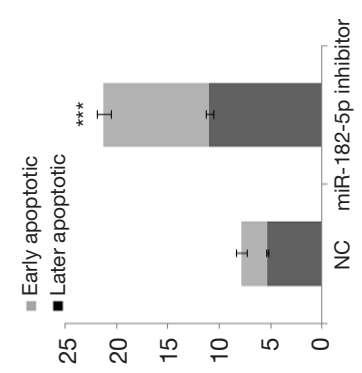

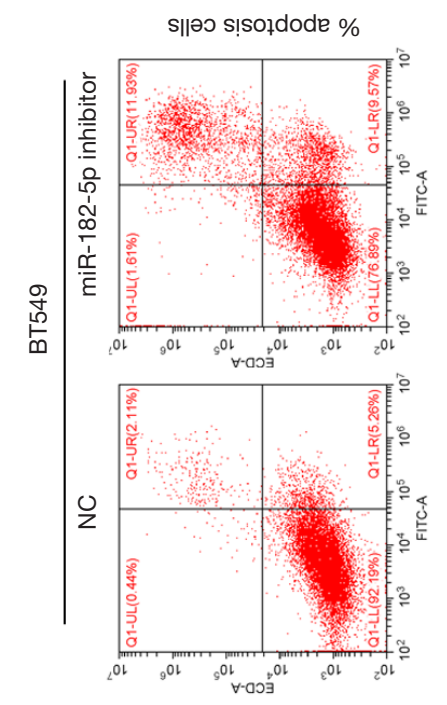

口
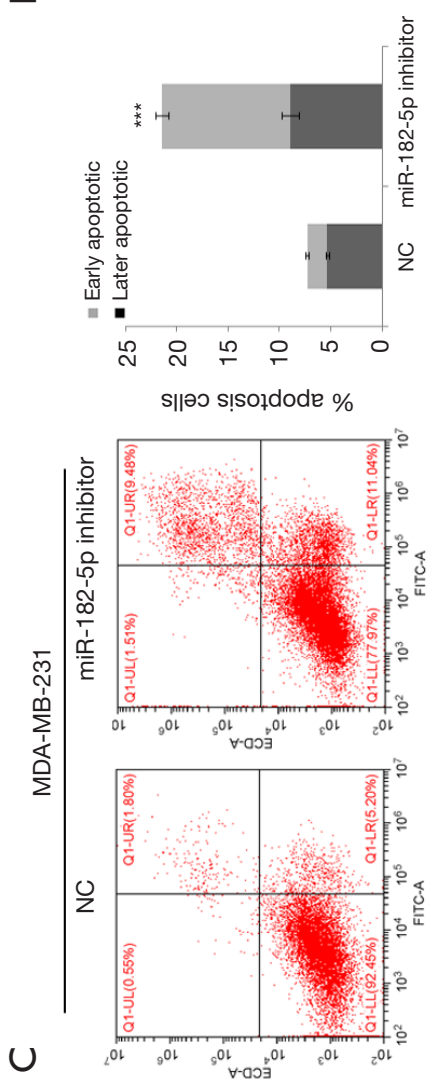

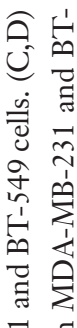

$\vec{\sim}: \Xi$

$\sum_{1}^{0}$ 至

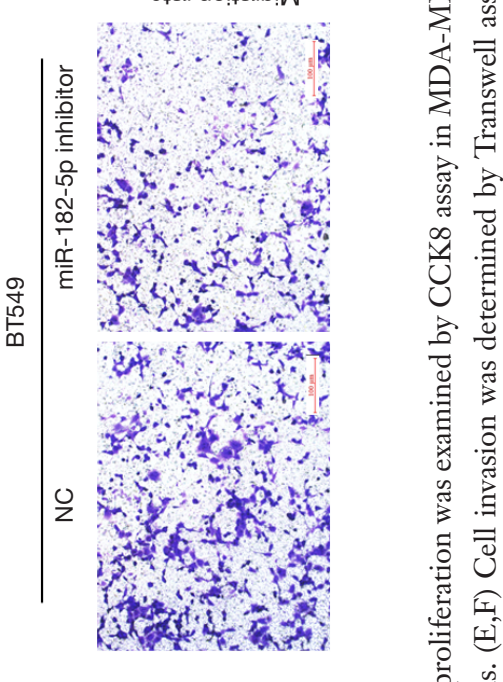

ᄂ

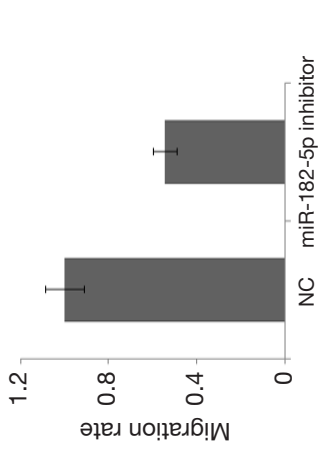

$\stackrel{\overline{2}}{\overline{0}} \frac{\infty}{8}$

ชิ

is

¿

党

$\stackrel{\widetilde{\pi}}{\sim}$

苂

की

总

길

is

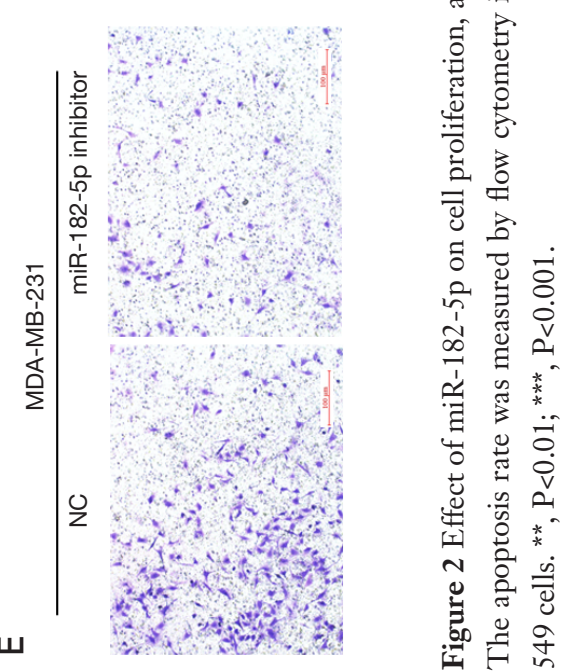



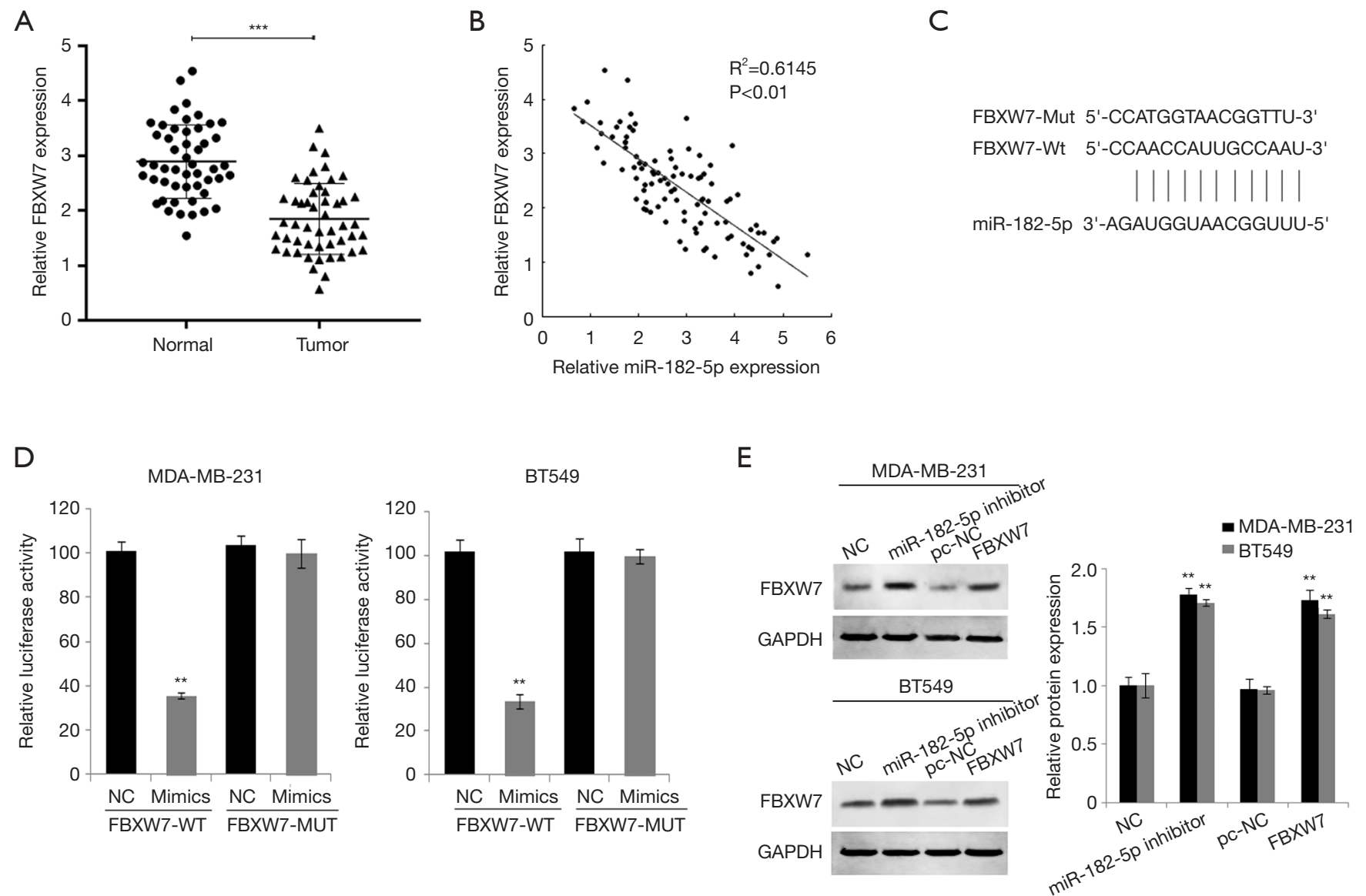

Figure 3 MiR-182-5p targets and negatively regulates FBXW7. (A) The expression of FBXW7 in TNBC tissues was examined by qRTPCR. (B) Pearson correlation analysis was performed to identify the correlation between miR-182-5p and FBXW in TNBC tissues. (C) The schematic diagram reflected the complementary binding sites between miR-182-5p and FBXW. (D) Dual-luciferase reporter gene assay showed the luciferase activity in TNBC cells. (E) The expression of FBXW7 in TNBC cells was measured by Western blot assay. ${ }^{* *}$, $\mathrm{P}<0.01$; ***, $\mathrm{P}<0.001$. TNBC, triple-negative breast cancer.

549 cells (Figure 4D). Transwell results confirmed that overexpression of FBXW7 notably inhibited the invasion rate of MDA-MB-231 (Figure 4E) and BT-549 (Figure 4F) cells. The above results suggested that FBXW7 may inhibit TNBC cell proliferation, invasion, and apoptosis.

\section{Downregulation of miR-182-5p inbibits TNBC cell proliferation and invasion and promotes cell apoptosis via FBXW7}

After co-transfecting TNBC cells with miR-182-5p inhibitor and si-FBXW7, we found that the down-regulation of FBXW7 expression reversed the inhibition of miR-182-5p expression on cell proliferation and invasion, and reversed the promotion of miR-182-5p expression down-regulation on cell apoptosis (Figure $5 A, B, C, D)$. The results suggested that miR-182-5p may play a regulatory role in tumor proliferation, apoptosis, and invasion by targeting FBXW7.

\section{Downregulation of miR-182-5p inbibits TLR4/NF- $\mathrm{KB}$ patbway activity in TNBC cells via FBXW7}

$F B X W 7$ gene is a p53-dependent tumor suppressor gene that takes part in the regulation of inflammation. Some studies have demonstrated that FBXW7 can inhibit inflammatory signal activity by downregulating the expression of $\mathrm{C} / \mathrm{EBP} \delta$ and its target gene TLR4 (3). In this study, we first detected the level of inflammatory cytokines in TNBC cell culture supernatant. ELISA results showed that the downregulation of miR-182-5p expression 

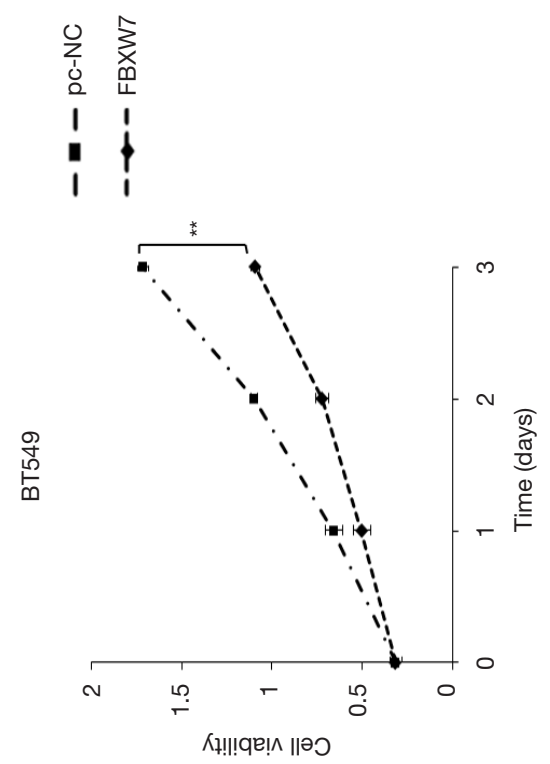

$\infty$
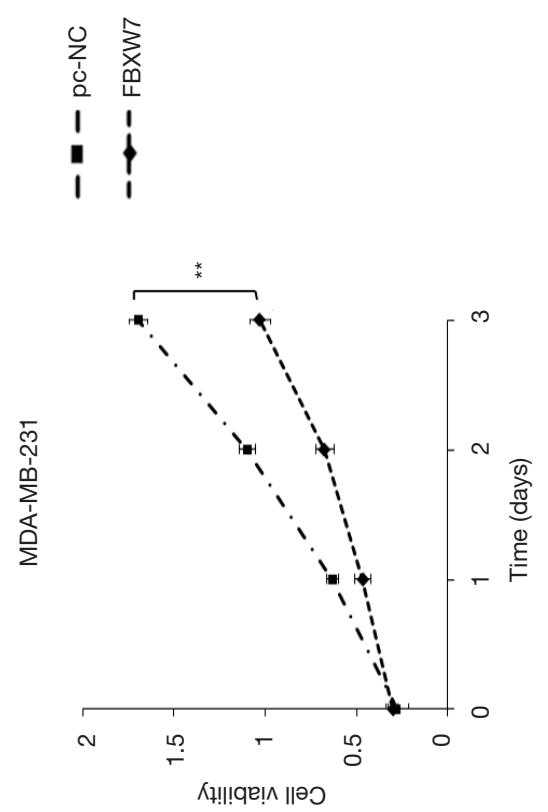

$\varangle$

(c) Annals of Translational Medicine. All rights reserved.
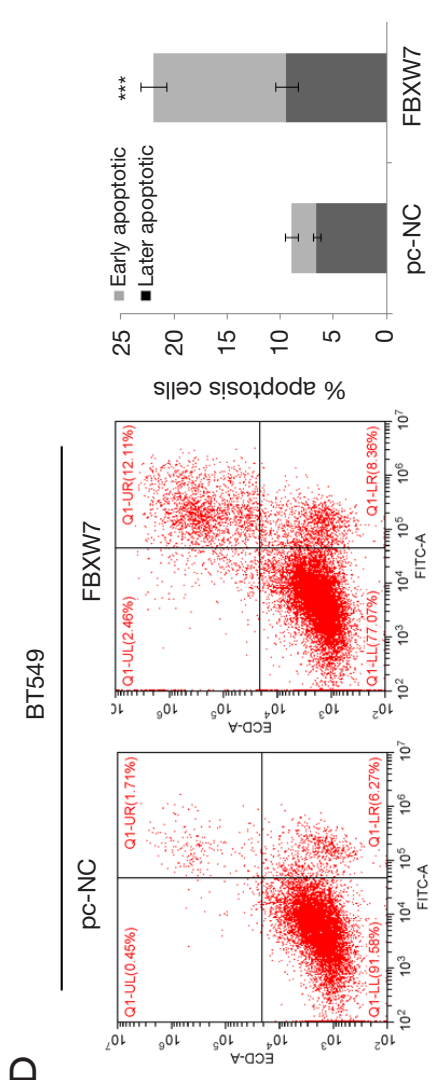

レ
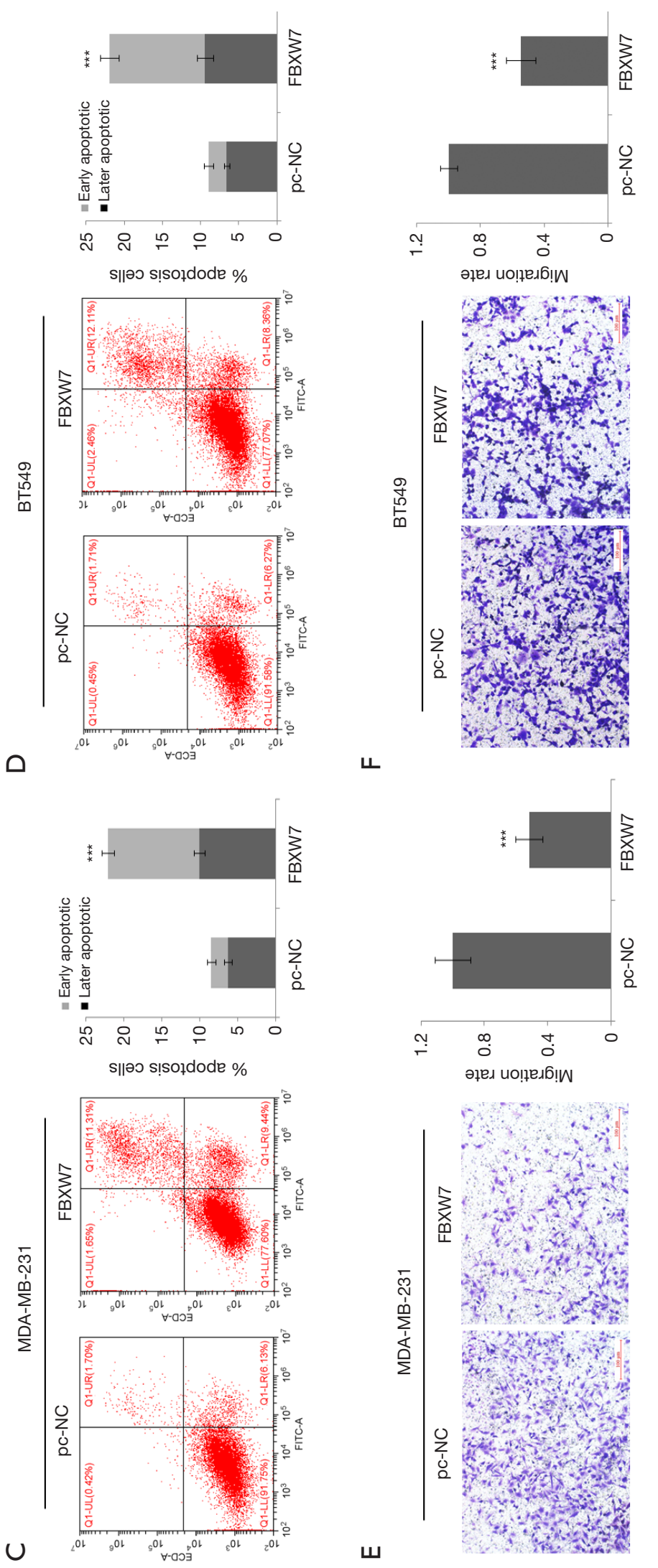

山

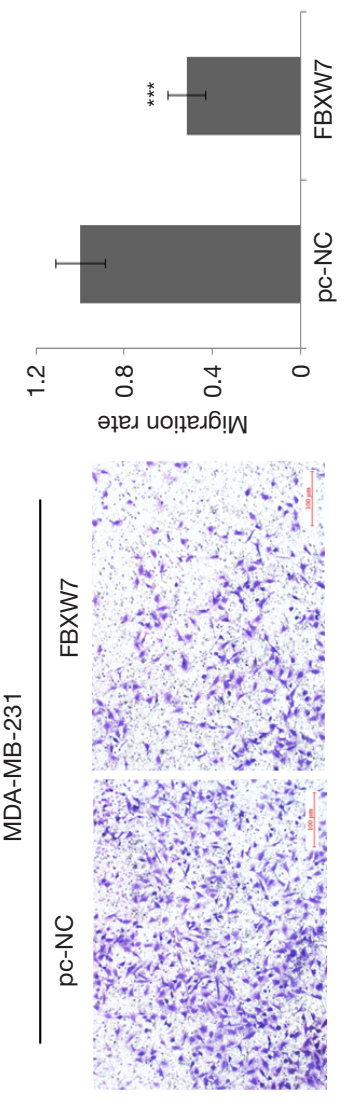



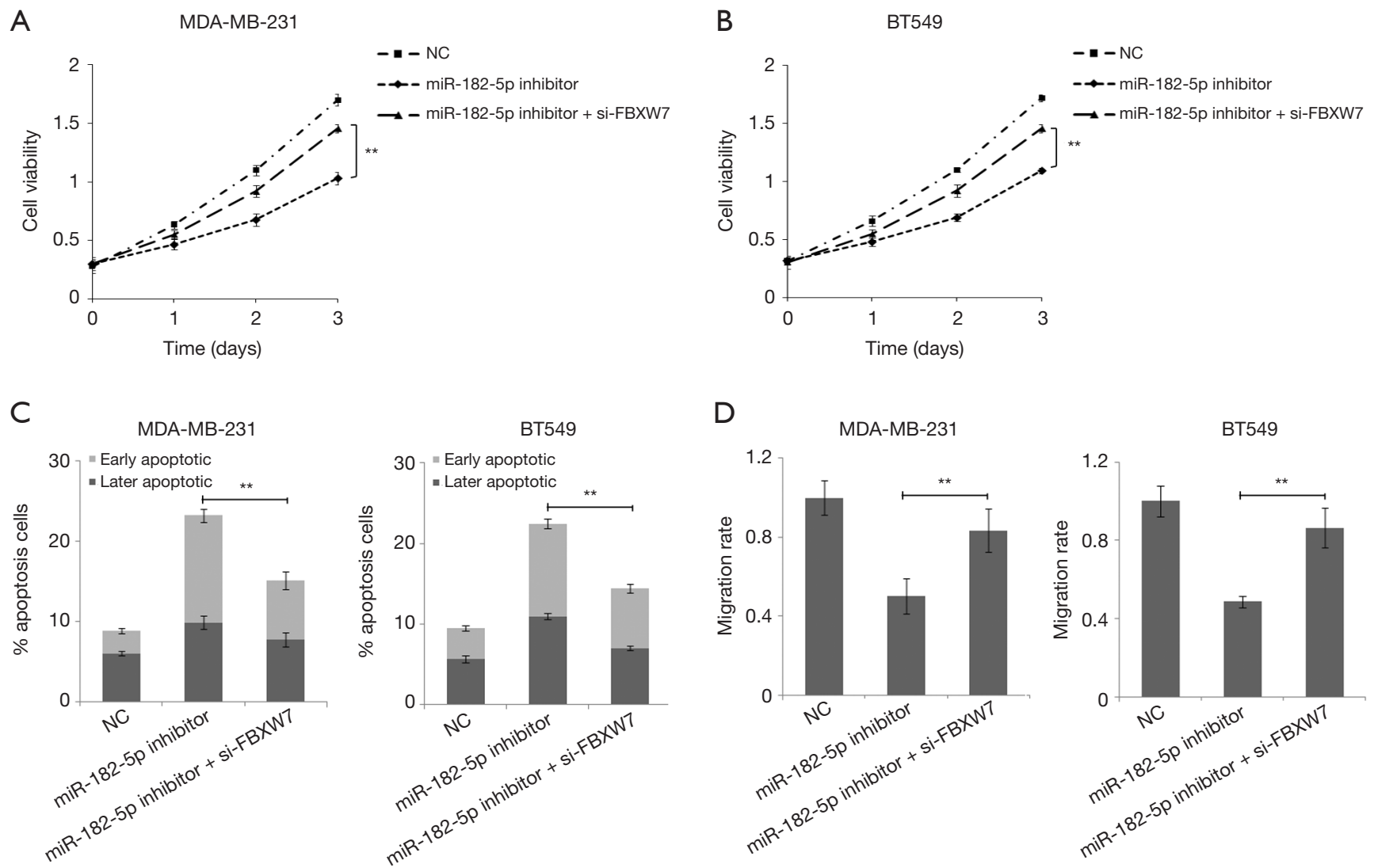

Figure 5 MiR-182-5p regulates TNBC cell proliferation, apoptosis, and invasion via FBXW. (A,B) Cell proliferation was examined by CCK8 assay in MDA-MB-231 and BT-549 cells. (C) The apoptosis rate was examined by flow cytometry in MDA-MB-231 and BT-549 cells. (D) Cell invasion was examined by Transwell assay in MDA-MB-231 and BT-549 cells. **, $\mathrm{P}<0.01$.

significantly reduced the levels of TNF- $\alpha$, IL- $1 \beta$, IL- 6 , and IL-18 in the supernatant of MDA-MB-231 and BT-549 cell cultures while inhibiting the expression of FBXW7 reversed the effect of miR-182-5p (Figure 6A,B). Further analysis by Western blot revealed that downregulation of miR-182$5 p$ remarkably reduced the expression of TLR4, MyD488, p-p65, and p-I-кB in MDA-MB-231 and BT-549 cells, while inhibition of FBXW7 significantly reversed the effect of miR-182-5p (Figure 6C,D). The above results indicated that the downregulation of miR-182-5p expression might inhibit the release of inflammatory factors and the activity of inflammatory pathways by up-regulating the expression of FBXW7, thus inhibiting the proliferation and invasion of TNBC cells and promoting cell apoptosis.

\section{Discussion}

TNBC, characterized by its highly aggressive and metastatic features, has higher recurrence and mortality than other types of BC due to insufficient effective treatment (22). MiRNA, as a new biomarker, plays a significant role in regulating the invasion, proliferation, metastasis, apoptosis, and chemotherapy of BC (23). In this study, we hypothesized that miR-182-5p participates in the regulation of TNBC cell proliferation and invasion and discussed the target and potential mechanism of miR-182-5p. We found that miR-182-5p was overexpressed in TNBC tissues and cells. Down-regulation of miR-182-5p expression can inhibit the proliferation and invasion of TNBC cells and promote cell apoptosis. These results suggested that miR182-5p may be a potential regulator in TNBC.

Through bioinformatics analysis, we predicted and verified the direct target FBXW7 of miR-182-5p, and the expression of the two in TNBC tissue was negatively correlated. $F B X W 7$ is a human tumor suppressor gene. It is reported that its total mutation rate in human tissues 

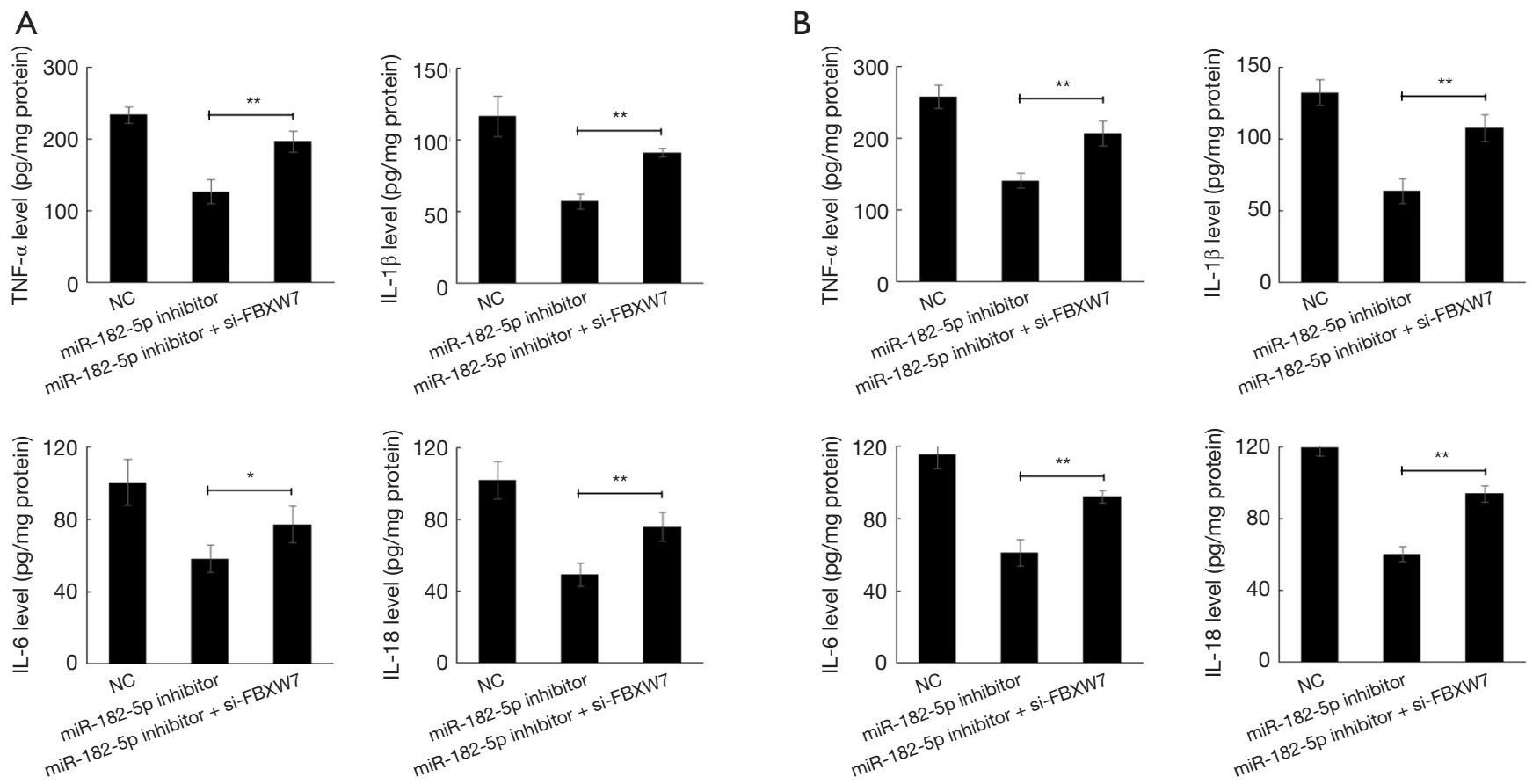

C
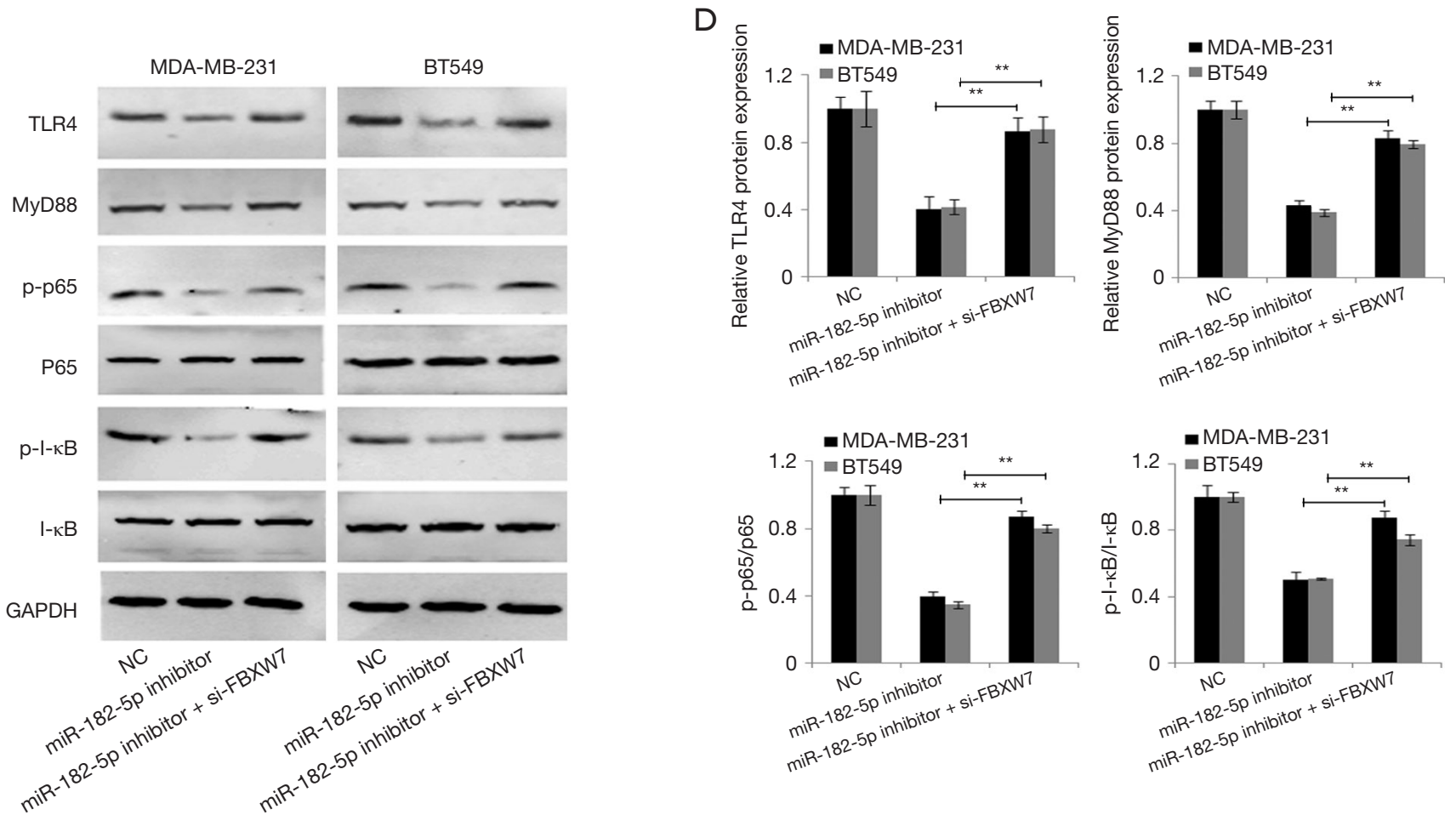

Figure 6 miR-182-5p regulates TLR4/NF- $\mathrm{kB}$ pathway activity in TNBC cells via FBXW7. (A,B) The levels of TNF- $\alpha$, IL-1 $\beta$, IL-6, and IL-18 in MDA-MB-231 and BT-549 cells were evaluated by ELISA assay. (C) The expression of the TLR4/NF-кB pathway in MDAMB-231 and BT-549 cells was examined by Western blot. (D) The quantitative diagram of the expression of the TLR4/NF-kB pathway. *, $\mathrm{P}<0.05 ;{ }^{* *}, \mathrm{P}<0.01$. TNBC, triple-negative breast cancer. 
including bile duct, blood, bone, brain, breast, colon, endometrium, stomach, lung, ovary, pancreas, and prostate is $6 \%$, and it can take part in the regulation of genetic instability or growth disorder of tumors by affecting ubiquitination and cycle of various tumor proteins $(24,25)$. Studies have confirmed down-regulation of FBXW7 expression promotes the proliferation of BC cells and inhibits cell apoptosis (26), whereas overexpression of FBXW7 can inhibit the proliferation of BC cells and promote cell apoptosis by targeting MTDH (27). In TNBC, FBXW7 loss of function can promote tumor growth and metastasis by stabilizing interferon receptor signals (28). In this study, we found that overexpression of FBXW7 significantly inhibited the proliferation and invasion of TNBC cells and promoted cell apoptosis. This result was consistent with earlier reports. Our further research showed that inhibiting FBXW7 expression can reverse the effect of miR-182-5p on TNBC cell proliferation, apoptosis, and invasion. The above results suggested that miR-182-5p regulates the proliferation, apoptosis, and invasion of TNBC cells through targeted negative regulation of FBXW7. However, the mechanism of miR-182-5p regulating TNBC cells via FBXW7 needs further research.

According to research, $F B X W 7$ can inhibit inflammatory signal activity on the one hand by downregulating the expression of $\mathrm{C} / \mathrm{EBP} \delta$ and its target gene TLR4. However, it is also inhibited by C/EBP $\delta$ and promotes tumor metastasis $(3,29)$. It is suggested that FBXW7 is strongly associated with inflammatory signals in the regulation of tumor progression. Therefore, we have detected the level of inflammatory signals in TNBC cells. The results indicated that the downregulation of miR$182-5 p$ expression significantly reduces the release level of inflammatory factors in TNBC cells, including TNF- $\alpha$, IL-1 $\beta$, IL-6, and IL-18, while inhibiting the expression of FBXW7 reverses the reduction of these inflammatory factors, indicating that miR-182-5p may regulate the proliferation, apoptosis, and invasion of TNBC cells by regulating the level of inflammatory signals. In this process, FBXW7 is the critical mediator of miR-182-5p regulation. A TLR4/NF- $\kappa$ B signaling pathway is widely reported taking part in the regulation of tumor progression and treatment through inflammatory reaction. For example, TLR4 can activate IL-4 receptor-related kinase 4 (IRAK4) and NF- $\kappa B$ by combining with paclitaxel or lipopolysaccharide, promote the production of IL-6, IL-8, vascular endothelial growth factor, and monocyte chemotactic protein-1, resist tumor cell apoptosis induced by chemotherapy drugs (30), and inhibit IL-6 and IL-8, thereby effectively reducing the chemotherapy drug resistance of BC mediated by TLR4 (7). In this study, we further detected the activity level of the TLR4/NF- $\mathrm{BB}$ pathway in TNBC cells. The results reflected that the downregulation of miR-182-5p significantly reduced the expression of TLR4, MyD488, p-p65, and p-I- $\kappa B$ in cells, while inhibition of FBXW7 significantly reversed the effect of miR-182-5p. This result supplied further evidence for the earlier conclusion.

In conclusion, this study proved that miR-182-5p participates in the regulation of TNBC cell proliferation, apoptosis, and invasion. The downregulation of miR$182-5 p$ inhibits the production of inflammatory factors and activation of inflammatory signals in TNBC cells by targeting FBXW7, thereby inhibiting the proliferation and invasion of TNBC cells and promoting cell apoptosis. This study will supply new therapeutic targets and the theoretical basis for TNBC treatment. Nevertheless, the role of MiR$182-5 \mathrm{p}$ in TNBC tumor growth and metastasis needs further study.

\section{Acknowledgments}

Funding: Study on the culture system of primary tumor tissue mass (201604020079).

\section{Footnote}

Reporting Checklist: The authors have completed the MDAR reporting checklist. Available at http://dx.doi.org/10.21037/ atm-20-5192

Data Sharing Statement: Available at http://dx.doi. org/10.21037/atm-20-5192

Conflicts of Interest: All authors have completed the ICMJE uniform disclosure form (available at http://dx.doi. org/10.21037/atm-20-5192). The authors have no conflicts of interest to declare.

Ethical Statement: The authors are accountable for all aspects of the work in ensuring that questions related to the accuracy or integrity of any part of the work are appropriately investigated and resolved. This study was approved by the Ethics Committee of Sun Yat-sen University Cancer Center (No. RDDA2020001590). 
Open Access Statement: This is an Open Access article distributed in accordance with the Creative Commons Attribution-NonCommercial-NoDerivs 4.0 International License (CC BY-NC-ND 4.0), which permits the noncommercial replication and distribution of the article with the strict proviso that no changes or edits are made and the original work is properly cited (including links to both the formal publication through the relevant DOI and the license). See: https://creativecommons.org/licenses/by-nc-nd/4.0/.

\section{References}

1. Garrido-Castro AC, Lin NU, Polyak K. Insights into Molecular Classifications of Triple-Negative Breast Cancer: Improving Patient Selection for Treatment. Cancer Discov 2019;9:176-98.

2. Wang M, Hu Y, Hou L, et al. A clinical study on the use of Huaier granules in post-surgical treatment of triplenegative breast cancer. Gland Surg 2019;8:758-765.

3. Wang RX, Xu XE, Huang L, et al. eEF2 kinase mediated autophagy as a potential therapeutic target for paclitaxelresistant triple-negative breast cancer. Ann Transl Med 2019;7:783.

4. Kwa MJ, Adams S. Checkpoint inhibitors in triple-negative breast cancer (TNBC): Where to go from here. Cancer 2018;124:2086-103.

5. Coussens LM, Werb Z. Inflammation and cancer. Nature 2002;420:860-67.

6. Zandi Z, Kashani B, Bashash D, et al. The anticancer effect of the TLR4 inhibition using TAK-242 (resatorvid) either as a single agent or in combination with chemotherapy: A novel therapeutic potential for breast cancer. J Cell Biochem 2020;121:1623-34.

7. Sootichote R, Thuwajit P, Singsuksawat E, et al. Compound A attenuates toll-like receptor 4-mediated paclitaxel resistance in breast cancer and melanoma through suppression of IL-8. BMC Cancer 2018;18:231.

8. Brown J, Wang H, Hajishengallis GN, et al. TLR-signaling networks: an integration of adaptor molecules, kinases, and cross-talk. J Dent Res 2011;90:417-27.

9. Kadowaki N, Ho S, Antonenko S, et al. Subsets of human dendritic cell precursors express different toll-like receptors and respond to different microbial antigens. J Exp Med 2001;194:863-9.

10. Ossovskaya V, Wang Y, Budoff A, et al. Exploring molecular pathways of triple-negative breast cancer. Genes Cancer 2011;2:870-9.

11. Baldwin AS. Control of oncogenesis and cancer therapy resistance by the transcription factor NF-kappaB. J Clin Invest 2001;107:241-6.

12. Bentires-Alj M, Barbu V, Fillet M, et al. NF-kappaB transcription factor induces drug resistance through MDR1 expression in cancer cells. Oncogene 2003;22:90-7.

13. Karin M. Nuclear factor-kappaB in cancer development and progression. Nature 2006;441:431-6.

14. Han SS, Yun H, Son DJ, et al. NF-kappaB/STAT3/PI3K signaling crosstalk in iMyc E mu B lymphoma. Mol Cancer 2010;9:97.

15. Liu C, Chen Z, Fang M, et al. MicroRNA let-7a inhibits proliferation of breast cancer cell by downregulating USP32 expression. Transl Cancer Res 2019;8:1763-71.

16. Li C, Zhang J, Ma Z, et al. miR-19b serves as a prognostic biomarker of breast cancer and promotes tumor progression through $\mathrm{PI} 3 \mathrm{~K} / \mathrm{AKT}$ signaling pathway. Onco Targets Ther 2018;11:4087-95.

17. Kong WQ, Bai R, Liu T, et al. MicroRNA-182 targets cAMP-responsive element-binding protein 1 and suppresses cell growth in human gastric adenocarcinoma. Febs J 2012;279:1252-60.

18. Sharifi M, Moridnia A. Apoptosis-inducing and antiproliferative effect by inhibition of miR-182-5p through the regulation of CASP9 expression in human breast cancer. Cancer Gene Ther 2017;24:75-82.

19. Paszek S, Gablo N, Barnas E, et al. Dysregulation of microRNAs in triple-negative breast cancer. Ginekol Pol 2017;88:530-6.

20. Hirata H, Ueno K, Shahryari V, et al. MicroRNA-182$5 \mathrm{p}$ promotes cell invasion and proliferation by down regulating FOXF2, RECK and MTSS1 genes in human prostate cancer. PLoS One 2013;8:e55502.

21. Zhao YS, Yang WC, Xin HW, et al. MiR-182-5p Knockdown Targeting PTEN Inhibits Cell Proliferation and Invasion of Breast Cancer Cells. Yonsei Med J 2019;60:148-57.

22. Yuan Z, Jiang H, Zhu X, et al. Ginsenoside Rg3 promotes cytotoxicity of Paclitaxel through inhibiting NFkappaB signaling and regulating $\mathrm{Bax} / \mathrm{Bcl}-2$ expression on triple-negative breast cancer. Biomed Pharmacother 2017;89:227-32.

23. Bertoli G, Cava C, Castiglioni I. MicroRNAs: New Biomarkers for Diagnosis, Prognosis, Therapy Prediction and Therapeutic Tools for Breast Cancer. Theranostics 2015;5:1122-43.

24. Mao JH, Kim IJ, Wu D, et al. FBXW7 targets mTOR for degradation and cooperates with PTEN in tumor suppression. Science 2008;321:1499-502. 
25. Akhoondi S, Sun D, von der Lehr N, et al. FBXW7/ hCDC4 is a general tumor suppressor in human cancer. Cancer Res 2007;67:9006-12.

26. Xia W, Zhou J, Luo H, et al. MicroRNA-32 promotes cell proliferation, migration and suppresses apoptosis in breast cancer cells by targeting FBXW7. Cancer Cell Int 2017;17:14.

27. Chen X, Li XY, Long M, et al. The FBXW7 tumor suppressor inhibits breast cancer proliferation and promotes apoptosis by targeting MTDH for degradation. Neoplasma 2018;65:201-9.

28. Singh S, Kumar S, Srivastava RK, et al. Loss of ELF5FBXW7 stabilizes IFNGR1 to promote the growth and

Cite this article as: $\mathrm{Wu} \mathrm{X}$, Chen $\mathrm{H}, \mathrm{Wu} M$, Peng S, Zhang L. Downregulation of $\mathrm{miR}-182-5 \mathrm{p}$ inhibits the proliferation and invasion of triple-negative breast cancer cells through regulating TLR4/NF- $\kappa$ B pathway activity by targeting FBXW7. Ann Transl Med 2020;8(16):995. doi: 10.21037/atm-20-5192 metastasis of triple-negative breast cancer through interferongamma signalling. Nat Cell Biol 2020;22:591-602.

29. Balamurugan K, Wang JM, Tsai HH, et al. The tumour suppressor C/EBPdelta inhibits FBXW7 expression and promotes mammary tumour metastasis. Embo J 2010;29:4106-17.

30. Szajnik M, Szczepanski MJ, Czystowska M, et al. TLR4 signaling induced by lipopolysaccharide or paclitaxel regulates tumor survival and chemoresistance in ovarian cancer. Oncogene 2009;28:4353-63.

(English Language Editor: J. Chapnick) 